SUPPORTING INFORMATION

FOR

\title{
An Unusual Occurrence on Attempted Purine C-8 Electrophilic Fluorination of 5'-Noraristeromycin
}

\author{
Atanu Roy and Stewart W. Schneller* \\ Department of Chemistry and Biochemistry, Auburn University, Auburn, Alabama \\ 36849-5312 \\ schnest@auburn.edu
}




\section{Experimental Section}

Materials and Methods. Melting points were recorded on a Meltemp II melting point apparatus and are uncorrected. ${ }^{1} \mathrm{H}$ and ${ }^{13} \mathrm{C}$ NMR spectra were recorded on a Bruker $\mathrm{AV}$ 400 spectrometer (operated at 400 or $100 \mathrm{MHz}$, respectively). All ${ }^{1} \mathrm{H}$ chemical shifts are reported in $\delta$ relative to internal standard tetramethylsilane (TMS, $\delta 0.00$ ). ${ }^{13} \mathrm{C}$ chemical shifts are reported in $\delta$ relative to $\mathrm{CDCl}_{3}$ (center of triplet, $\delta$ 77.23) or relative to DMSO$d_{6}$ (center of septet, $\delta 39.51$ ). The spin multiplicities are indicated by the symbols $\mathrm{s}$ (singlet), d (doublet), $\mathrm{t}$ (triplet), q (quartet), $\mathrm{m}$ (multiplet) and br (broad), dd (doublet of doublet). The optical rotation was carried out on a Jasco P1010 polarimeter and the ultraviolet spectra recorded using a Hitachi U-2000 spectrophotometer. Elemental analysis were performed by Atlantic Microlabs, Atlanta, Georgia. Reactions were monitored by thin layer chromatography (TLC) using $0.25 \mathrm{~mm}$ E. Merck silica gel 60$\mathrm{F}_{254}$ precoated silica gel plates with visualization by irradiation with a Mineral light UVGL-25 lamp or exposure to iodine vapor. Column chromatography was performed on Whatman silica gel (average particle size 5-25 $\mu \mathrm{m}, 60 \AA$ ) and elution with the indicated solvent system. Yields refer to chromatographically and spectroscopically $\left({ }^{1} \mathrm{H}\right.$ and ${ }^{13} \mathrm{C}$ NMR) homogeneous materials. The reactions were generally carried out in a $\mathrm{N}_{2}$ atmosphere under anhydrous conditions.

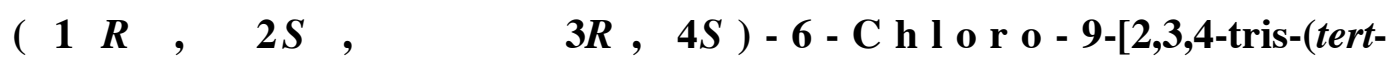
butyldimethylsilanyloxy)cyclopentyl]-9H-purine (4). Compound $\mathbf{3}^{12}$ (3.28 g, 8.52 mmol) was dissolved in DMF (60 mL) followed by addition of tert-butyldimethylsilyl chloride (4.5 g, $29.85 \mathrm{mmol}, 3.5$ equiv.) and imidazole (4.0 g, $58.75 \mathrm{mmol}, 6.9$ equiv.). The resulting solution was stirred at room temperature for $24 \mathrm{~h}$. The mixture was poured 
into $\mathrm{Et}_{2} \mathrm{O}$-ice cold $\mathrm{H}_{2} \mathrm{O}(100 \mathrm{~mL}, 2: 1)$ and the organic layer was separated, dried $\left(\mathrm{Na}_{2} \mathrm{SO}_{4}\right)$ and evaporated to dryness. Column chromatographic purification $($ EtOAc-hexanes $=1: 9)$ of the residue provided 4 (4.95 g, 95\%) as a colorless liquid: $\mathrm{UV}\left(\mathrm{CH}_{2} \mathrm{Cl}_{2}\right) \lambda_{\max } 250 \mathrm{~nm}(\varepsilon$ 415.3); ${ }^{1} \mathrm{H}$ NMR $\left(\mathrm{CDCl}_{3}, 400 \mathrm{MHz}\right) \delta$ - 0.64 (s, 3H), - 0.27 (s, 3H), 0.08 (s, 3H), 0.10 (s, $3 \mathrm{H}), 0.11(\mathrm{~s}, 3 \mathrm{H}), 0.12(\mathrm{~s}, 3 \mathrm{H}), 0.65(\mathrm{~s}, 9 \mathrm{H}), 0.90(\mathrm{~s}, 9 \mathrm{H}), 0.93(\mathrm{~s}, 9 \mathrm{H}), 1.82(\mathrm{dd}, 1 \mathrm{H}, J=$ 4.32, $14.76 \mathrm{~Hz}), 2.87-2.95(\mathrm{~m}, 1 \mathrm{H}), 3.82(\mathrm{t}, 1 \mathrm{H}, J=1.71 \mathrm{~Hz}), 4.04(\mathrm{~d}, 1 \mathrm{H}, J=4.88 \mathrm{~Hz})$, $4.67(\mathrm{dd}, 1 \mathrm{H}, J=3.28,7.72 \mathrm{~Hz}), 5.04-5.10(\mathrm{~m}, 1 \mathrm{H}), 8.33(\mathrm{~s}, 1 \mathrm{H}), 8.70(\mathrm{~s}, 1 \mathrm{H}) ;{ }^{13} \mathrm{C}$ NMR $\left(\mathrm{CDCl}_{3}, 100 \mathrm{MHz}\right) \delta-5.6,-4.7,-4.6,-4.5,-4.4,-4.3,17.9,18.1,18.2,25.8,25.9,26.0$, 38.8, 58.9, 75.4, 79.2, 80.4, 131.7, 144.9, 151.1, 152.0, 152.4. Anal. Calcd. for $\mathrm{C}_{28} \mathrm{H}_{53} \mathrm{ClN}_{4} \mathrm{O}_{3} \mathrm{Si}_{3}: \mathrm{C}, 54.82 ; \mathrm{H}, 8.71 ; \mathrm{N}, 9.13$. Found: C, 54.92; H, 8.85; N, 9.03.

$(1 R, 2 S, 3 R, 4 S)$ - 8-Benzenesulfonyl-6-chloro-9-[2,3,4-tris-(tertbutyldimethylsilanyloxy)cyclopentyl]-9H-purine (5). $n$-Butyllithium (4.9 mL, 2.5 M, $12.24 \mathrm{mmol}, 3$ equiv.) was added dropwise to a stirred solution of $\mathbf{4}(2.5 \mathrm{~g}, 4.08 \mathrm{mmol})$ in dry THF $(50 \mathrm{~mL})$ at $-78{ }^{\circ} \mathrm{C}$ under a nitrogen atmosphere. The mixture was stirred for 1.5 $\mathrm{h}$ at $-78{ }^{\circ} \mathrm{C}$. To this, a solution of N-fluorobenzenesulfonimide $(3.86 \mathrm{~g}, 12.24 \mathrm{mmol}, 3$ equiv.) in dry THF (20 mL) was added and the mixture was stirred further for $3 \mathrm{~h}$. The reaction was then quenched by the addition of saturated aq $\mathrm{NH}_{4} \mathrm{Cl}(70 \mathrm{~mL})$ and the solution extracted with EtOAc, dried $\left(\mathrm{Na}_{2} \mathrm{SO}_{4}\right)$ and evaporated to dryness. The residue was purified via flash chromatography on silica gel eluting with EtOAc-hexanes (1:9) to afford $2.4 \mathrm{~g}$ (78 \%) of 5 as a gum: UV (DMSO) $\lambda_{\max } 310 \mathrm{~nm}(\varepsilon 510.6) ;{ }^{1} \mathrm{H}$ NMR $\left(\mathrm{CDCl}_{3}\right.$, $400 \mathrm{MHz}) \delta-0.65$ (s, 3H), -0.28 (s, 3H), 0.07 (s, 3H), 0.08 (s, 3H), 0.09 (s, 3H), 0.10 (s, 3H), 0.64 (s, 9H), 0.90 (s, 9H), 0.93 (s, 9H), 1.78-1.83 (m, 1H), 2.86-2.94 (m, 1H), 3.81 $(\mathrm{s}, 1 \mathrm{H}), 4.00(\mathrm{~d}, 1 \mathrm{H}, J=4.88 \mathrm{~Hz}), 5.60(\mathrm{q}, 1 \mathrm{H}, J=8.96 \mathrm{~Hz}), 5.83(\mathrm{dd}, 1 \mathrm{H}, J=3.2,9.44$ 
$\mathrm{Hz}), 7.52(\mathrm{t}, 2 \mathrm{H}, J=7.64 \mathrm{~Hz}), 7.63(\mathrm{t}, 1 \mathrm{H}, J=7.48 \mathrm{~Hz}), 8.08(\mathrm{~d}, 2 \mathrm{H}, J=7.76 \mathrm{~Hz}), 8.68$ (s, $1 \mathrm{H}) ;{ }^{13} \mathrm{C}$ NMR $\left(\mathrm{CDCl}_{3}, 100 \mathrm{MHz}\right) \delta$-6.6, -5.8, -5.7, -5.6, -5.5, -5.4, 16.8, 17.0, 17.1, 24.7, 24.8, 24.9, 37.8, 57.9, 75.4, 79.2, 80.4, 128.7 (2C), 129.6 (2C), 132.5, 134.4, 139.5, 145.4, 151.5, 152.4, 152.8. Anal. Calcd. for $\mathrm{C}_{34} \mathrm{H}_{57} \mathrm{ClN}_{4} \mathrm{O}_{5} \mathrm{SSi}_{3}: \mathrm{C}, 54.19 ; \mathrm{H}, 7.62 ; \mathrm{N}$, 7.43. Found: C, $54.02 ; \mathrm{H}, 7.69 ; \mathrm{N}, 7.38$.

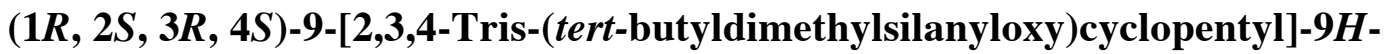
purin-6-ylamine (14). A solution of $4(4.55 \mathrm{~g}, 7.42 \mathrm{mmol})$ in saturated methanolic ammonia $(100 \mathrm{~mL})$ was sealed in a Parr stainless stell vessel and heated at $120{ }^{\circ} \mathrm{C}$ for 22 h. After cooling to room temperature, the solvent was evaporated to dryness and the residue purified via flash chromatography using $\mathrm{Et}_{2} \mathrm{O} /$ hexanes (1:1) to give $\mathbf{1 4}$ (4.32 g, 98\%) as a white solid: $\mathrm{mp} 70{ }^{\circ} \mathrm{C}$; $\mathrm{UV}\left(\mathrm{CH}_{2} \mathrm{Cl}_{2}\right) \lambda_{\max } 250 \mathrm{~nm}\left(\varepsilon\right.$ 418.0); ${ }^{1} \mathrm{H} \mathrm{NMR}\left(\mathrm{CDCl}_{3}\right.$, $400 \mathrm{MHz}) \delta-0.56(\mathrm{~s}, 3 \mathrm{H}),-0.30(\mathrm{~s}, 3 \mathrm{H}), 0.05$ (s, 3H), 0.07 (s, 3H), 0.08 (s, 3H), 0.09 (s, 3H), $0.66(\mathrm{~s}, 9 \mathrm{H}), 0.87(\mathrm{~s}, 9 \mathrm{H}), 0.91(\mathrm{~s}, 3 \mathrm{H}), 1.73(\mathrm{dd}, 1 \mathrm{H}, J=4.16,14.64 \mathrm{~Hz}), 2.83-2.90$ (m, 1H), $3.80(\mathrm{t}, 1 \mathrm{H}, J=1.64 \mathrm{~Hz}), 3.98(\mathrm{~d}, 1 \mathrm{H}, J=4.36 \mathrm{~Hz}), 4.62(\mathrm{dd}, 1 \mathrm{H}, J=3.36,7.6$ $\mathrm{Hz}), 4.97-5.02(\mathrm{~m}, 1 \mathrm{H}), 6.60$ (brs, $2 \mathrm{H}), 8.01(\mathrm{~s}, 1 \mathrm{H}), 8.30(\mathrm{~s}, 1 \mathrm{H}) ;{ }^{13} \mathrm{C} \mathrm{NMR}\left(\mathrm{CDCl}_{3}, 100\right.$ MHz) $\delta$-5.6, -4.8, -4.7, -4.6, -4.5, -4.4, 17.9, 18.0, 18.1, 25.8, 25.9, 26.0, 39.3, 57.8, 75.5, 79.3, 80.6, 119.5, 139.7, 150.7, 152.8, 156.1. Anal. Calcd. for $\mathrm{C}_{28} \mathrm{H}_{55} \mathrm{~N}_{5} \mathrm{O}_{3} \mathrm{Si}_{3}$ : C, 56.61; H, 9.33; N, 11.79. Found: C, 56.23; H, 9.51; N, 11.50.

$(1 R, 2 S, 3 R, 4 S)$ - 8 - B e n z e n e s u l f o n y l - 9-[2,3,4-tris-(tertbutyldimethylsilanyloxy)cyclopentyl]-9H-purin-6-ylamine (15). Employing the same ammonolysis procedure that provided 14, compound $5(1.5 \mathrm{~g}, 2.0 \mathrm{mmol})$ afforded $1.26 \mathrm{~g}$ (86.3\%) of $\mathbf{1 5}$ as a white solid compound. Compound $\mathbf{1 5}$ was also prepared directly from 14 as follows: to a solution of diisopropylamine $(1.77 \mathrm{~mL}, 12.65 \mathrm{mmol}, 5$ equiv. $)$ in dry 
THF $(20 \mathrm{~mL})$ at $-20^{\circ} \mathrm{C}$, butyllithium $(5.1 \mathrm{~mL}, 2.5 \mathrm{M}, 12.65 \mathrm{mmol}, 5$ equiv.) was added under a nitrogen atmosphere. The resulting solution was stirred for $30 \mathrm{~min}$ and the temperature then taken to $-78{ }^{\circ} \mathrm{C}$. A solution of $\mathbf{1 4}(1.5 \mathrm{~g}, 2.53 \mathrm{mmol})$ in dry THF (20 $\mathrm{mL}$ ) was added dropwise with a syringe. The mixture was stirred for $1.5 \mathrm{~h}$ at $-78{ }^{\circ} \mathrm{C}$. A solution of N-fluorobenzenesulfonimide ( $2.40 \mathrm{~g}, 7.59 \mathrm{mmol}, 3$ equiv.) in dry THF (20 $\mathrm{mL}$ ) was added and the mixture stirred further for $3 \mathrm{~h}$. Following this, the solution was quenched by the addition of saturated aq $\mathrm{NH}_{4} \mathrm{Cl}$ solution $(60 \mathrm{~mL})$ and the solution extracted with EtOAc, dried $\left(\mathrm{Na}_{2} \mathrm{SO}_{4}\right)$, and the solvent removed. The residue was purified by silica gel column chromatography eluting with EtOAc-hexanes (3:7) to afford $0.85 \mathrm{~g}$ (46\%) of 15 as a white solid (along with $0.45 \mathrm{~g}(30 \%)$ of starting material, 14): $\mathrm{mp} 150$ ${ }^{0} \mathrm{C}$; UV (DMSO) $\lambda_{\max } 310 \mathrm{~nm}\left(\varepsilon\right.$ 502.8); ${ }^{1} \mathrm{H}$ NMR (CDCl $\left.3,400 \mathrm{MHz}\right) \delta-0.60(\mathrm{~s}, 3 \mathrm{H}),-$ $0.46(\mathrm{~s}, 3 \mathrm{H}), 0.02(\mathrm{~s}, 3 \mathrm{H}), 0.08(\mathrm{~s}, 3 \mathrm{H}), 0.11(\mathrm{~s}, 3 \mathrm{H}), 0.16(\mathrm{~s}, 3 \mathrm{H}), 0.50(\mathrm{~s}, 9 \mathrm{H}), 0.95$ (s, $9 \mathrm{H}), 1.05(\mathrm{~s}, 9 \mathrm{H}), 1.96-2.03(\mathrm{~m}, 1 \mathrm{H}), 2.44-2.52(\mathrm{~m}, 1 \mathrm{H}), 3.81(\mathrm{~s}, 1 \mathrm{H}), 3.91(\mathrm{~d}, 1 \mathrm{H}, J=$ $6.08 \mathrm{~Hz}), 5.59(\mathrm{q}, 1 \mathrm{H}, J=9.12 \mathrm{~Hz}), 5.81(\mathrm{dd}, 1 \mathrm{H}, J=2.92,9.32 \mathrm{~Hz}), 7.22(\mathrm{brs}, 2 \mathrm{H}), 7.53$ $(\mathrm{t}, 2 \mathrm{H}, J=7.68 \mathrm{~Hz}), 7.63(\mathrm{t}, 1 \mathrm{H}, J=7.08 \mathrm{~Hz}), 8.08(\mathrm{~d}, 2 \mathrm{H}, J=8.0 \mathrm{~Hz}), 8.28(\mathrm{~s}, 1 \mathrm{H}) ;{ }^{13} \mathrm{C}$ NMR $\left(\mathrm{CDCl}_{3}, 100 \mathrm{MHz}\right) \delta-4.8,-4.7,-4.6,-4.5,-4.4,-4.3,17.8,17.9,18.1,25.5,25.6$, 25.8, 36.5, 60.8, 73.5 (2C), 79.1, 119.6, 128.7 (2C), 129.6 (2C), 134.4, 139.5, 147.8, 151.1, 154.5, 157.2. Anal. Calcd. for $\mathrm{C}_{34} \mathrm{H}_{59} \mathrm{~N}_{5} \mathrm{O}_{5} \mathrm{SSi}_{3}: \mathrm{C}, 55.62 ; \mathrm{H}, 8.10 ; \mathrm{N}, 9.54$. Found: C, 55.41; H, 8.22; N, 9.31.

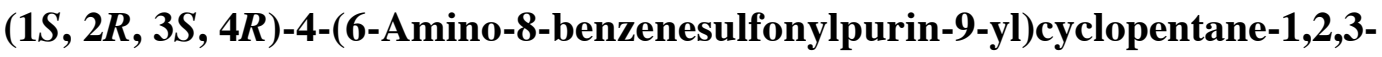
triol (2). Ammonium fluoride (912 $\mathrm{mg}, 24.6 \mathrm{mmol}$ ) was added to a solution of $\mathbf{1 5}$ (600 $\mathrm{mg}, 0.82 \mathrm{mmol})$ in $\mathrm{MeOH}(70 \mathrm{~mL})$ and the mixture was stirred at room temperature for $72 \mathrm{~h}$. After evaporation of the solvent, the resulting residue was purified by silica gel 
column chromatography $(\mathrm{MeOH}-\mathrm{EtOAc}=1: 9)$ to give $2(240 \mathrm{mg}, 76 \%): \mathrm{mp} 235^{\circ} \mathrm{C}$; $[\alpha]_{\mathrm{D}}^{23.7} 14.75(c, 0.24, \mathrm{DMSO})$; UV (DMSO) $\lambda_{\max } 320 \mathrm{~nm}\left(\varepsilon\right.$ 534.7); ${ }^{1} \mathrm{H}$ NMR (DMSO- $d_{6}$, $400 \mathrm{MHz}) \delta 1.93-2.00(\mathrm{~m}, 1 \mathrm{H}), 2.20-2.26(\mathrm{~m}, 1 \mathrm{H}), 3.78(\mathrm{dd}, 1 \mathrm{H}, J=3.45,7.80 \mathrm{~Hz}), 3.83$ $(\mathrm{d}, 1 \mathrm{H}, J=6.10 \mathrm{~Hz}), 4.84(\mathrm{~d}, 1 \mathrm{H}, J=6.14 \mathrm{~Hz}), 4.86(\mathrm{~d}, 1 \mathrm{H}, J=5.41 \mathrm{~Hz}), 4.95(\mathrm{q}, 1 \mathrm{H}, J=$ $5.64 \mathrm{~Hz}), 5.40(\mathrm{q}, 1 \mathrm{H}, J=8.51 \mathrm{~Hz}), 5.46(\mathrm{~d}, 1 \mathrm{H}, J=6.10 \mathrm{~Hz}), 7.70(\mathrm{t}, 2 \mathrm{H}, J=7.82 \mathrm{~Hz})$, $7.80(\mathrm{t}, 1 \mathrm{H}, J=7.34 \mathrm{~Hz}), 7.78-7.98(\mathrm{br}, 2 \mathrm{H}), 8.03(\mathrm{~d}, 2 \mathrm{H}, J=7.90 \mathrm{~Hz}), 8.23(\mathrm{~s}, 1 \mathrm{H}) ;{ }^{13} \mathrm{C}$ NMR (DMSO- $\left.d_{6}, 100 \mathrm{MHz}\right) \delta 34.8,60.8,72.4,73.6,76.5,118.8,128.0$ (2C), 129.8 (2C), 134.8, 138.9, 145.6, 149.8, 154.6, 157.3. Anal. Calcd. for $\mathrm{C}_{16} \mathrm{H}_{17} \mathrm{~N}_{5} \mathrm{O}_{5} \mathrm{~S}: \mathrm{C}, 49.10 ; \mathrm{H}$, 4.38; N, 17.89. Found: C, 49.41; H, 4.37; N, 18.14. 